\title{
Discussion on Construction Technology of Road Subgrade in Civil En- gineering
}

\author{
De Zhang*
}

Shanghai Qilu Civil Engineering Co., Ltd. E-mail: zhangde@163.com

\begin{abstract}
With the rapid development of cities, more and more new roads are built in various civil engineering projects. Subgrade construction is the core of road engineering system. Only by comprehensively ensuring the quality of road subgrade can the road performance be effectively improve and the development of urban construction be ensured. The quality of subgrade determines the traffic capacity of urban roads and plays a decisive role in the safety and stability of traffic. This article mainly analyzes the problems of road subgrade construction in civil engineering, and comprehensively puts forward countermeasures, so as to effectively improve the subgrade construction quality and ensure the smooth operation of roads.
\end{abstract}

Keywords: Civil Engineering; Roads; Traffic Capacity; Solum

With the rapid development of economy, China's urbanization process is getting faster and faster. In the process of urban development, civil engineering construction projects are very important, among which road subgrade construction is a very important content and a key link in various construction. Only by comprehensively ensuring the quality of subgrade construction can the smoothness, stability and safety of traffic be guaranteed. The quality of subgrade projects determines the quality of roads, so attention should be paid to it.

\section{Research on the construction technology of road subgrade in civil engineering}

\subsection{Measurement work in construction}

Scientific measurement must be carried out in construction, both in the early stage and in the process of construction. Scientific measurement data should be taken as guidance. The measurement should be based on design drawings, and the effective key data should be extracted. By measuring the site, the design ideas of drawings should be fully restored in the site, so that the drawings can be further confirmed in the site and it is conducive to comprehensively understanding the overall structure of the construction and local cognition. Good measurement is helpful for the restoration and adjustment of the road centerline. Through scientific and reasonable measurement, the outline, coordinates and size of the structure are completely marked, and the precise position is found, which provides reference for the rapid construction in the later period. Subgrade leveling points, center lines and traverse lines need to be measured with emphasis, which are the key contents and need to be comprehensively planned and carefully measured to ensure the accuracy and effectiveness for later construction. Then, surveyors need to grasp several problems. First, they should be fami-

This is an open-access article distributed under the terms of the Creative Commons Attribution Non-Commercial License (http://creativecommons.org/licenses/by-nc/4.0/), which permits unrestricted non-commercial use, distribution, and reproduction in any medium, provided the original work is properly cited. 
liar with the idea of drawing design, fully grasp the contents of drawings, and make measurements through the contents of drawings. Second, they should measure all operations in strict accordance with the requirements and drawings to ensure consistency with the drawing design. Third, it is necessary to look for possible omissions, and strictly carry out audit and inspection to ensure the accuracy of data. According to the needs of engineering construction, temporary leveling points and ground elevation should be set for re-measurement of the center line, so that the position of the cross section can be determined and the cross-sectional position can be ensured to meet the requirements of construction. In this way, the measuring, setting-out and positioning can be accurate.

\subsection{Fill subgrade construction technology}

Filling is an important link. If it is not well controlled, it will lead to roadbed sinking, affecting the quality of traffic and burying potential safety hazards. When filling, local materials and natural soil are used to fill subgrade soil in construction. In order to ensure the quality of subgrade filling, it is required to test the natural soil before filling to ensure that the soil meets the filling standard. Strictly check the properties of natural soil, and grasp the filling properties by checking the soil water content and mechanical properties. In order to improve the fill quality, it is necessary to measure the filled road sections, master the road conditions, and do a good job of filling test in advance. Only by drawing correct conclusions can the construction be carried out, and the overall quality can be fully guaranteed to meet the construction requirements. Several aspects should be paid attention to in road section test, such as reasonable arrangement of construction equipment, sparse index of test sample soil, and compaction times. Subgrade filling should be carried out according to the process, and be in accordance with construction standards. Layered construction should be carried out according to soil structure, and the thickness at different levels should be reasonably controlled. The thickness is below $30 \mathrm{~cm}$, the highest is $30 \mathrm{~cm}$, the compacted thickness of the last layer is not more than $8 \mathrm{~cm}$, and the lateral width of the layer is higher than the height of subgrade, which is above $50 \mathrm{~cm}$.

\subsection{Excavation subgrade construction tech- nology}

Excavation construction of subgrade is also important. Before excavation, it is necessary to fully understand the basic situation of subgrade construction. Before construction, it is necessary to understand the current soil structure of excavation, and grasp the soil condition to ensure the safety of construction. Scientific and reasonable analysis of soil quality is the premise to ensure safe construction. It is necessary to classify the soil quality strictly according to the construction standards, and determine the anti-leakage methods. In order to ensure the excavation quality, it is also required to clean up the ground and dig out the surface, and reserve intercepting ditches and drains to ensure smooth drainage. Subgrade slope should ensure stability and avoid surrounding buildings from being affected by construction. According to the basic requirements of design, carefully reading the drawing requirements is necessary. Excavators and dump trucks are used as transportation tools. If the geology is not suitable for the use of equipment and machinery, manpower is needed for special areas.

\subsection{Subgrade drainage and protection tech- nology}

Subgrade drainage construction is mainly to ensure a longer service life of roads, and to reduce subgrade water erosion and improve road use effect through good drainage settings. When setting up drainage, first, it is necessary to comprehensively treat large-area accumulated water. Water pump drainage can be used for drainage, or ditches can be dug for drainage according to terrain conditions to ensure rapid drainage of accumulated water in large areas. Second, surface water should be treated strictly. Rapids and ditches are mainly used, so that the surface water can be removed quickly and the construction progress and quality can be avoided. The third is scientific prevention and control of groundwater. It is very important to clear know the groundwater level. Different drainage methods should be selected according to different water levels, and blind ditch, underground ditch and deep well are generally used for drainage. Subgrade protection should use cold-resistant vegetation with developed roots to ensure stability. In addition, concrete construction can be adopted according to different road sections to ensure stable structure.

\section{Main problems}




\subsection{Low strength of soft soil}

Subgrade, as the focus of municipal road construction, needs strict construction standards to ensure the overall quality and safety. At present, there are soft soil layers under many roadbeds, which brings difficulty to construction. When constructing in soft soil layers, the surrounding soil layers are easily affected by vibration or extrusion, which reduces the strength, resulting in deformation and sinking of the pavement.

\subsection{Rain erosion of slope subgrade}

It is common for the subgrade of municipal road slope to be washed by rain, which is also the key problem at present. If it is not handled well, the road surface will collapse. The ability of road slope subgrade to resist rain erosion determines the service life of road.

\subsection{Difficulty in controlling the proportion of subgrade settlement and residual settlement}

Municipal planning and design are complex, and it is necessary to fully grasp the structure of the whole road section. When designing, it is necessary to do a scientific and rigorous design for the more complex road section, so as to fully ensure the subgrade strength and hardness. It is necessary to improve the soil hardness and ensure the medium-term strength through scientific methods, and it is impossible to accurately grasp the proportion of subgrade settlement and residual settlement, which will cause subgrade quality problems and affect safe passage.

\section{Improvement measures}

\subsection{Do a good job of measurement before construction}

Construction survey can provide the basis for scientific construction in an all-round way, so it is necessary to pay full attention to all the work of subgrade construction survey, divide key areas and directions, ensure the accuracy of traverse, centerline and leveling points, and retest the survey results, which need accurate data support from whole to local. In order to ensure the measurement quality, it is necessary to carefully check the instrument before measurement to ensure the accuracy of the instrument. Relevant technical personnel should be aware of the drawings, avoid measurement loopholes, affect the construction quality, check the unreasonable places of the drawings, eliminate various unfavorable factors, and measure the subgrade. The re-measurement of the center line is mainly to set the appropriate ground elevation and temporary leveling base point elevation. Although the measurement process will be affected, it must be reasonably controlled within the standard range to ensure the positioning accuracy of the vertical and horizontal sections. Underground facilities and pipe network need to be taken into account during measurement to avoid unnecessary losses.

\subsection{Reasonably treat inferior soil}

Inferior soil is not conducive to construction, so it is necessary to comprehensively treat and improve soil properties to ensure the safety and stability of subgrade. There are three ways to treat inferior soil. First, to organize the construction according to the soil conditions. In order to improve the construction efficiency and ensure the construction progress, it is necessary to divide the structure reasonably, measure the soil properties on site, use mechanical equipment according to different structural properties, and make full use of the method of combining mechanical equipment with manpower to construct the road section. The second is to use modern technology for processing. Different soil properties lead to different treatment methods. Chemical materials such as lime and cement can be used properly according to different properties to improve soil strength and avoid swelling. The third is to use physical means. Physical methods can also be used to treat inferior soil. By heating and pressurizing the soil, the structural strength of the soil can be further improved, the original properties of the soil can be changed, and the stability of the subgrade can be comprehensively improved.

\subsection{Apply fill improvement technology}

The quality of filling affects the life of subgrade, so it is necessary to do a good job of filling in an all-round way, and take measures to solve different problems, so as to further improve the soil properties of subgrade and ensure the rapid completion of filling. In general, soil stiffening can be used to improve soil stability. Generally, synthetic materials will be used. Before construction, it is necessary to fully grasp the subgrade conditions. During the construction, composite materials will be buried in the pavement to further improve the strength and reduce the subgrade pressure and stress. Using composite materials, the subgrade foundation pit caused by pavement rolling 
can be effectively reduced. Soil properties can also be improved by increasing soil shear strength.

\subsection{Do a good job of subgrade drainage}

Road construction is greatly affected by weather and environment, so it is necessary to comprehensively deal with rain and snow. First, to scientifically and rationally layout the subgrade surface drainage facilities. Build an effective drainage system, so that the ground drainage can be quickly removed. There are generally two methods: centralized drainage and decentralized drainage. Centralized drainage is to set up transverse slopes and side ditches to remove rainwater, and scattered drainage can be carried out in flat areas. The second is to revise the surface drainage import and export. Existing facilities shall be repaired and used to effectively avoid scouring, blocking, freezing and other phenomena and ensure smooth ground water flow. Third, to reinforce the subgrade slope. Increasing vegetation, mortar rubble, hanging net and spray protection can improve the slope strength in an all-round way, and retaining wall can also ensure the foundation safety. Fourth, retaining walls should be set at drainage gates. In this way, it can effectively improve the waterproof ability and achieve the reinforcement effect can be achieved. It can solve the problem of rainwater gravity drainage, reduce the damage of subgrade can be reduced, and avoid the poor drainage or underwater seepage of road surface, thus further improving the safety of pavement and subgrade.

\section{Conclusion}

The stability of municipal road subgrade is related to the safety of pavement, so it is necessary to do a good job in the construction of key links. It is required to improve the quality of subgrade through scientific and technical methods, and choose the most economical and effective subgrade construction scheme while ensuring the lowest cost, so as to comprehensively improve the economic and social benefits of municipal roads.

\section{References}

1. Wang G. Research on road engineering construction in civil engineering (in Chinese). China Housing Facilities 2019; (4): 83-84.

2. An Y, Chen Q, Yue J. Research on teaching content of tunnel engineering in civil engineering under large civil engineering (in Chinese). Cai Zhi 2018; (7): 55.

3. Zhuge Y. Research on the construction of road engineering in civil engineering (in Chinese). Traffic World 2017; 441(27): 46-47. 